\title{
CircRNA LRIG3 knockdown inhibits the progression of hepatocellular carcinoma by regulating miR-223- 3p/MAP2K6 axis and inactivating MAPK/ERK pathway
}

Hui Sun

Laiyang Central Hospital of Yantai City

Junwei Zhai

Shandong Tengzhou Central People's Hospital

\section{Li Zhang}

Heze Jiazheng Vocational College

Yingnan Chen ( $\nabla$ sunhuish1111@163.com )

Shandong Tengzhou Central People's Hospital

\section{Research}

Keywords: hepatocellular carcinoma, circ_LRIG3, miR-223-3p, MAP2K6, MAPK/ERK pathway

Posted Date: March 17th, 2020

DOI: https://doi.org/10.21203/rs.3.rs-17512/v1

License: (c) (1) This work is licensed under a Creative Commons Attribution 4.0 International License.

Read Full License 


\section{Abstract}

Background: Emerging evidence suggests that circular RNAs (circRNAs) play critical roles in tumorigenesis. However, the roles and molecular mechanism of circRNA leucine-rich repeat immunoglobulin domain-containing protein 3 (circ_LRIG3) in hepatocellular carcinoma (HCC) have not been investigated.

Methods: The expression levels of circ_LRIG3, microRNA-223-3p (miR-223-3p), and mitogen-activated protein kinase kinase 6 (MAP2K6) were determined by quantitative real-time polymerase chain reaction (qRT-PCR). Flow cytometry was applied to determine the cell cycle distribution and cell apoptosis. Cell proliferation, migration and invasion were assessed by methylthiazolyldiphenyl-tetrazolium bromide (MTT) assay and transwell assay, respectively. Western blot assay was employed to measure the protein levels of snail, E-cadherin, MAP2K6, mitogen-activated protein kinase (MAPK), phospho-MAPK (p-MAPK), extracellular signal-regulated kinases (ERKs), and phospho-ERKs ( $p$-ERKs). The relationship between miR223-3p and circ_LRIG3 or MAP2K6 was predicted by bioinformatics tools and verified by dual-luciferase reporter assay. A xenograft tumor model was established to confirm the functions of circ_LRIG3 in vivo.

Results: Circ_LRIG3 and MAP2K6 expression were enhanced while miR-223-3p abundance was reduced in HCC tissues and cells. Knockdown of circ_LRIG3 inhibited the progression of HCC cells via reducing cell proliferation, metastasis and increasing apoptosis. MiR-223-3p was a target of circ_LRIG3 and its downregulation reversed the inhibitory effect of circ_LRIG3 knockdown on progression of HCC cells. Moreover, MAP2K6 could bind to miR-223-3p, and MAP2K6 upregulation also abolished the suppressive impact of circ_LRIG3 interference on progression of HCC cells. Additionally, silence of circ_LRIG3 suppressed the activation of MAPK/ERK pathway and tumor growth by upregulating miR-223-3p and downregulating MAP2K6.

Conclusion: Circ_LRIG3 knockdown inhibited HCC progression through regulating miR-223-3p/MAP2K6 axis and inactivating MAPK/ERK pathway, providing a potential therapeutic approach for patients with HCC.

\section{Background}

Liver cancer is one of the most lethal and prevalent cancers worldwide, causing approximately 745,500 deaths in 2012 [1]. It has been reported that hepatocellular carcinoma (HCC) is the main histological subtype of liver cancer and may cause huge economic burden [2]. Despite great advancement in therapeutic approaches, including surgery, chemotherapy and radiation therapy, the overall survival rate for advanced the patients with HCC is very poor [3]. Therefore, it is critical to clarify the underlying mechanisms of HCC progression and search new therapeutic strategies.

As a new type of endogenous non-coding RNA, circular RNA (circRNA) has become a research hotspot in RNA field and attracted widespread attention [4]. Unlike linear RNAs, circRNAs have covalently closedloop structures with neither 5' cap nor 3' polyadenylated tail and not easily affected by RNA exonuclease 
and more stable than linear RNAs $[5,6]$. Up to now, some studies have shown that circRNAs are extensively expressed in many types of cells and participated in the progression and development of diverse cancers, including HCC $[7,8]$. For instance, circRNA Cdr1as served as an oncogene in HCC via regulating miR-7 expression [9]. CircRNA cSMARCA5 could restrain HCC cell growth and metastasis [10]. In addition, circRNA leucine-rich repeat and immunoglobulin domain-containing protein 3 (circ_LRIG3; hsa_circ_0027345, chr12:59277301-59308117) has been reported to be overexpressed in HCC tissues [11]. Nevertheless, the functional roles and molecular mechanism of circ_LRIG3 in HCC progression have not been clarified.

It is widely acknowledged that circRNAs can modulate gene expression via acting as miRNA sponges in eukaryotes, which is one of main mechanisms of physiological and pathological processes [12]. MicroRNAs (miRNAs), a class of non-coding RNAs ( 22 nucleotides), play regulatory roles in disease through interaction with mRNAs [13]. MiR-223 has been identified to play an anti-cancer role in HCC and it might be a possible therapeutic target for treating HCC [14]. However, the connection between circ_LRIG3 and miR-223-3p has not been reported. It has been suggested that mitogen-activated protein kinase kinase 6 (MAP2K6) can serve as a critical regulator in promoting tumorigenesis [15]. However, the role of MAP2K6 in HCC cells has not been reported. In our research, we first investigated the associations among miR-223-3p, circ_LRIG3 and MAP2K6 in HCC cells.

Here, we measured miR-223-3p, circ_LRIG3 and MAP2K6 expression in HCC tissues and cells, and determined their functions in HCC cells. Besides, we probed the circ_LRIG3/miR-223-3p/MAP2K6 regulatory network in the progression of HCC. The aim of our study was to offer a new insight into the diagnosis and treatment of HCC.

\section{Materials And Methods}

\section{Specimens collection}

In our research, HCC tissues $(n=46)$ and adjacent normal tissues $(n=46)$ were acquired from patients who underwent surgery at Laiyang Central Hospital Of Yantai City. These tissues were harvested and timely frozen in liquid nitrogen, and then preserved at $-80^{\circ} \mathrm{C}$ until the experiments were performed. These subjects did not receive any treatment and provided informed consents.

\section{Cell culture and transfection}

Human HCC cell lines (Hep3B and Huh7) and human normal liver cell line (THLE-2) were bought from COBIOER (Nanjing, China). These cells were grown in Dulbecco's modified eagle medium (DMEM; Hyclone, Logan, Utah, USA) supplemented with $10 \%$ fetal bovine serum (FBS; Gibco, Carlsbad, CA, USA) in a moist atmosphere with $5 \%$ carbon dioxide at $37^{\circ} \mathrm{C}$.

Small interfering RNA against circ_LRIG3 (si-circ_LRIG3) and its matched control (si-NC), MAP2K6 overexpression plasmid (MAP2K6) and its matched control (pcDNA) were synthesized by RIBOBIO 
(Guangzhou, China). Mimic or inhibitor of miR-223-3p (miR-223-3p or anti-miR-223-3p) and mimic or inhibitor negative control (miR-NC or anti-miR-NC) were provided by GenePharma (Jiangsu, China). Lentivirus-mediated shRNA interference targeting circ_LRIG3 (sh-circ_LRIG3) and its matched control (shNC) were bought from Genechem (Shanghai, China). Lipofectamine 3000(Invitrogen, Carlsbad, CA, USA) was used for cell transfection.

\section{Quantitative real-time polymerase chain reaction (qRT-PCR)}

Trizol reagent (Invitrogen) was utilized to obtain total RNA from tissue samples and cells. For detecting genes expression, Prime Script RT reagent Kit (Takara, Dalian, China) and TaqMan MicroRNA Reverse Transcription Kit (Thermo Fisher Scientific, Waltham, MA, USA) were used to synthesize the first strand of complementary DNA (cDNA). All reactions were performed on the ABI 7300 system (Thermo Fisher Scientific) using SYBR Green PCR kit (Thermo Fisher Scientific). Primers for circ_LRIG3, LRIG3, miR-2233p, MAP2K6, U6, and glyceraldehyde-3-phosphate dehydrogenase (GAPDH) were exhibited as followed: circ_LRIG3 forward (F, 5'- TCACTGGTTTGGATGCATTG-3'; R, 5'-AAGGTGGCTCATGGAACTTG-3'), LRIG3 (F, 5'-CACATCAATGGAACCTGGGTATTTTGAC-3'; R, 5'- GTTTCGGTTCAATTCGAGATGTTGCAGTT-3'), miR223-3p (F, 5'- AGCTGGTGTTGTGAATCAGGCCG-3'; R, 5'-TGGTGTCGTGGAGTCG-3'), MAP2K6, (F, 5'ATTTGGAGTCTGGGCATCAC-3'; R, 5'- ACTTGTCTGCTGGGAGTTGTG-3'), GAPDH (F, 5'CGCTCTCTGCTCCTCCTGTTC-3'; R, 5'-ATCCGTTGACTCCGACCTTCAC-3'), U6 (F, 5'CTCGCTTCGGCAGCACATATACT-3'; R, 5'-ACGCTTCACGAATTTGCGTGTC-3'). The circ_LRIG3, LRIG3, MAP2K6, or miR-223-3p expression was assessed using the $2^{-\triangle \triangle C t}$ method and standardized by GAPDH or U6, respectively.

\section{RNase R and Actinomycin D treatment}

To assess the stability of circ_LRIG3 and its linear isoform (LRIG3), dimethyl sulfoxide solution (DMSO) or actinomycin $D(2 \mathrm{mg} / \mathrm{mL})$ was added to cultured medium. RNase $\mathrm{R}(3 \mathrm{U} / \mu \mathrm{g}$, Epicentre Technologies, Madison, WI, USA) was utilized to incubate the total RNA $(2 \mu \mathrm{g})$ at $37^{\circ} \mathrm{C}$ for $30 \mathrm{~min}$. After treatment with RNase R or Actinomycin D, these cells were collected and then subjected to qRT-PCR for detecting the expression levels of circ_LRIG3 and LRIG3.

\section{Subcellular fractionation location}

PARIS Kit (Life Technologies Corp., Grand Island, NY, USA) was employed to isolate cytosolic and nuclear fractions. In brief, Hep3B and Huh7 cells were carefully washed by phosphate-buffered saline (PBS) and placed on the ice. Subsequently, these cells were re-suspended in fractionation buffer and centrifuged at $500 \times \mathrm{g}$ at $4^{\circ} \mathrm{C}$ for $5 \mathrm{~min}$. Subsequently, the cytoplasmic fraction would be separated from the nuclear pellet. After that, the remaining nuclear pellet was again lysed by cell disruption buffer as nuclear fraction. Lastly, the abundance of U6, GAPDH and circ_LRIG3 was examined by qRT-PCR in the nuclear and cytoplasmic fractions. GAPDH and U6 were served as controls for the cytoplasmic and nuclear, respectively. 


\section{Cell cycle assay}

Hep3B and Huh7 cells were collected following transfection for $48 \mathrm{~h}$, and fixed by ice-cold ethanol (70\%) at $-20^{\circ} \mathrm{C}$ for $24 \mathrm{~h}$. Afterward, these cells were centrifuged and washed with PBS, followed by staining with $25 \mu \mathrm{g} / \mathrm{mL}$ propidium iodide (PI) solution in PBS supplemented with Triton X-100 (0.2\%) and RNase $\mathrm{A}(50 \mu \mathrm{g} / \mathrm{mL})$ for $20 \mathrm{~min}$ in the dark. Lastly, flow cytometry (Guava Technologies, Hayward, CA, USA) was employed to examine the distribution of cell cycle.

\section{Cell proliferation assay}

Cell proliferation ability was evaluated using methylthiazolyldiphenyl-tetrazolium bromide (MTT) assay. In short, Hep3B and Huh7 cells were placed in the 96-well plates overnight and then transfected with siNC, si-circ_LRIG3, si-circ_LRIG3 + anti-miR-NC, si-circ_LRIG3 + anti-miR-223-3p, si-circ_LRIG3 + pcDNA, or si-circ_LRIG3 + MAP2K6. MTT solution $(20 \mu \mathrm{L}, 5 \mathrm{mg} / \mathrm{mL}$, Sangon Biotech, Shanghai, China) was added to per well following transfection for $0 \mathrm{~h}, 24 \mathrm{~h}, 48 \mathrm{~h}$, or $72 \mathrm{~h}$. Following incubation for $4 \mathrm{~h}$ at $37^{\circ} \mathrm{C}$, DMSO $(150 \mu \mathrm{L})$ was added to per well after removing the cultured medium. The absorbance of per well was examined with the microplate reader (Bio-Teck, Winooski, VT, USA) at $490 \mathrm{~nm}$.

\section{Cell apoptosis assay}

Annexin V-fluorescein isothiocyanate (FITC)/PI apoptosis detection kit (Sangon Biotech) was applied to detect cell apoptosis according to the recommendations. In short, Hep3B and Huh7 cells were harvested and double stained by Annexin V-FITC and PI for $20 \mathrm{~min}$ in the darkness. Afterward, apoptotic cells were detected using a flow cytometer.

\section{Transwell assay}

Transwell chambers (pore size $8 \mu \mathrm{m}$ ) (Corning Incorporation, Corning, NY, USA) coated without and with Matrigel (BD Biosciences, San Jose, CA, USA) were utilized to assess Hep3B and Huh7 cell migration and invasion abilities, respectively. In brief, cells were suspended in serum-free medium (DMEM, $100 \mu \mathrm{L}$ ) and then placed in the top surface of the chamber, and DMEM mixed with FBS $(10 \%)$ was placed in the bottom surface of the chamber. Non-migrated or non-invaded cells from the top surface were gently wiped off using a cotton wool after incubation for $24 \mathrm{~h}$. After that, the migrated or invaded cells were fixed using paraformaldehyde (4\%) and stained using crystal violet (0.1\%). Lastly, a microscope (Olympus, Tokyo, Japan) was utilized to photograph and count the migrated and invaded cells.

\section{Western blot assay}

To extract the total protein, tissues or transfected cells were lysed by RIPA lysis buffer (Sigma-Aldrich, St. Louis, MO, USA) containing 1mM phenylmethylsulphonyi fluoride (PMSF; Sigma-Aldrich). After quantification by using BCA protein assay kit (Thermo Fisher Scientific), protein (about $40 \mu \mathrm{g}$ ) was resolved by sodium dodecyl sulfate-polyacrylamide gel electrophoresis (SDS-PAGE). Afterwards, polyvinylidene fluoride (PVDF; Beyotime, Shanghai, China) membranes were applied to transfer the 
protein. Next, membranes would be blocked using 5\% skim milk (Yili, Beijing, China) for $1 \mathrm{~h}$, and then the membranes were probed with specific primary antibody against snail (1:500, ab180714, Abcam, Cambridge, MA, USA), E-cadherin (1:500, ab15148, Abcam), MAP2K6 (1:2000, ab154901, Abcam), mitogen-activated protein kinase (MAPK) (1:1000, ab236738, Abcam), phospho-MAPK (p-MAPK) (1:500, ab47363, Abcam), extracellular signal-regulated kinases (ERKs) (1:1000, ab17942, Abcam) phosphorERKs (p-ERKs) (1:1000, ab47339, Abcam), or GAPDH (1:2000, ab37168, Abcam) overnight at $4{ }^{\circ} \mathrm{C}$. After that, these membranes were incubated by horseradish peroxidase (HRP)-conjugated goat anti-rabbit immunoglobulin (lg) G (1:4000, ab205718, Abcam). Finally, all protein bands were observed with the enhanced chemiluminescence reagent (Tanon, Shanghai, China). Relative protein expression was quantified by ImageJ software, followed by normalizing to GAPDH expression.

\section{Dual-luciferase reporter assay}

Circinteractome or TargetScan software online was utilized to predict the potential binding sites of miR223-3p and circ_LRIG3 or MAP2K6. The circ_LRIG3 or MAP2K6 3'UTR fragments containing wild-type (WT; containing the specific binding site with miR-223-3p) or mutant (MUT; harboring the mutational binding sits with miR-223-3p) were amplified and cloned into pmirGIO luciferase reporter vector (Promega, Madison, WI, USA), namely WT vectors (circ_LRIG3-wt, MAP2K6-wt) or MUT vectors (circ_LRIG3-mut, MAP2K6-mut). Hep3B and Huh7 cells were co-transfected with those reporter vectors and miR-223-3p (or miR-NC) for 48 h. Lastly, dual-luciferase assay system (Promega) was utilized for detecting the luciferase activity, followed by normalizing to Renilla luciferase activity.

\section{In vivo tumor model}

The sh-circ_LRIG3 or sh-NC was transfected into Huh7 cells. Stably transfected cells $\left(2 \times 10^{6}\right)$ were injected subcutaneously into BALB/c nude mice ( $n=6 /$ group, male, six-week-old, Shanghai Experimental Animal Center, Shanghai, China). From the 7th day, tumor length and width were examined with a caliper every week and tumor volumes (length $\times$ width $^{2} \times 0.5$.) were calculated. After injection for 4 weeks, these mice would be sacrificed and tumor specimens were weighed and collected for further analysis.

\section{Statistical analysis}

In this study, all data from at least three independently experiment were displayed as mean \pm standard deviation (SD). The significance of differences between groups was analyzed with Student's $t$-test (for 2 groups) or a one-way analysis of variance (ANOVA; for more than 2 groups). Correlation between miR223-3p and circ_LRIG3 or MAP2K6 was detected by Spearman rank correlation. Statistical analyses were performed by Graphpad Prism version 6.0 software (GraphPad Software, San Diego California, USA). $P<0.05$ was considered to be a statistically significant difference.

\section{Results}

\section{Circ_LRIG3 expression was increased in HCC tissues and cells}


To investigate the potential roles of circ_LRIG3 in HCC, its expression was examined in HCC tissues and cells by qRT-PCR. Results displayed that circ_LRIG3 level was strikingly enhanced in HCC tissues in comparison with normal tissues (Figure 1A). Similarly, its expression was also increased in HCC cells (Hep3B and Huh7) compared with THLE-2 cells (Figure 1B). Next, we evaluated the stability of circ_LRIG3 in HCC cells. According to the qRT-PCR analysis, circ_LRIG3 was resistant to RNase R relative to linear LRIG3 in Hep3B and Huh7 cells (Figure 1C and 1D), implying that circ_LRIG3 formed a loop structure. Subsequently, Actinomycin D assay demonstrated that the half-life of circ_LRIG3 transcript exceeded 24 h, indicating that circ_LRIG3 transcript was more stable than the linear LRIG3 transcript in Hep3B and Huh7 cells (Figure 1E and 1F). Moreover, the localization of circ_LRIG3 was analyzed in HCC cells. As presented in Figure $1 \mathrm{G}$ and $1 \mathrm{H}$, most of the circ_LRIG3 was located in the cytoplasm. These results suggested that circ_LRIG3 might play critical roles in the progression of HCC.

\section{Knockdown of circ_LRIG3 inhibited cell proliferation, metastasis and induced apoptosis in HCC cells}

To explore the effects of circ_LRIG3 on proliferation, metastasis and apoptosis of HCC cells, si-NC or sicirc_LRIG3 was transfected into Hep3B and Huh7 cells. The results from qRT-PCR analysis showed that the expression of circ_LRIG3 was evidently reduced in Hep3B and Huh7 cells after transfection with sicirc_LRIG3, suggesting that transfection of si-circ_LRIG3 was successful (Figure 2A). Cell cycle progression was analyzed by flow cytometry and cell proliferation was determined by MTT assay. Results displayed that the percentage of G0/G1 phase cells was increased by downregulating circ_LRIG3, while the percentage of cells in $\mathrm{S}$ and G2/M phases were reduced after interference of circ_LRIG3 (Figure $2 B$ and $2 D$ ), suggesting that the cell cycle was arrested at the G0/G1 phase. And MTT analysis proved that cell proliferation was obviously inhibited in Hep3B and Huh7 cells transfected with si-circ_LRIG3 compared with those cells transfected with si-NC (Figure 2C and 2E). Moreover, we found that cell apoptosis was enhanced in Hep3B and Huh7 cells transfected with si-circ_LRIG3 in contrast to sh-NC group (Figure 2F). Transwell assay showed that interference of circ_LRIG3 inhibited Hep3B and Huh7 cell migration and invasion (Figure $2 \mathrm{G}$ and $2 \mathrm{H}$ ). Western blot assay was applied to measure the metastasisrelated proteins (snail and E-cadherin). As depicted in Figure $2 \mathrm{I}$ and 2J, circ_LRIG3 silence decreased the protein level of snail (a mesenchymal marker) while increased the protein expression of E-cadherin (an epithelial marker) in Hep3B and Huh7 cells. These data collectively indicated that downregulation of circ_LRIG3 could inhibit the progression of HCC cells.

\section{MiR-223-3p was a direct target of circ_LRIG3}

Previous study indicated that circRNAs could act as molecular sponges of miRNAs in HCC cells [16], so the possible target miRNAs of circ_LRIG3 were predicted by circinteractome tool. As shown in Figure 3A, miR-223-3p was predicted as a target of circ_LRIG3. To investigate whether miR-223-3p was a direct target of circ_LRIG3, we performed dual-luciferase reporter assay in HCC cells. Results showed that transfection of miR-223-3p mimic resulted in a significant reduction in luciferase activity of circ_LRIG3-wt compared to miR-NC group, while the luciferase activity of circ_LRIG3-mut was unaffected by transfection of miR-223-3p (Figure 3B and 3C). Next, we explored the impact of circ_LRIG3 on miR-223-3p 
expression. The results of qRT-PCR demonstrated that transfection of si-circ_LRIG3 led to an obvious promotion of miR-223-3p expression, while co-transfection of anti-miR-223-3p abated this effect (Figure 3D). Subsequently, the expression of miR-223-3p was detected by qRT-PCR in HCC tissues and cells. As illustrated in Figure 3E and 3F, the expression of miR-223-3p was downregulated in HCC cells and tissues compared with their corresponding controls. Moreover, correlation between miR-223-3p and circ_LRIG3 expression was analyzed in HCC tissues. As displayed in Figure 3G, miR-223-3p expression was negatively correlated with circ_LRIG3 level in HCC tissues $(r=-0.5054, P=0.0003)$. Thus, these results demonstrated that miR-223-3p was a target of circ_LRIG3 in HCC cells.

\section{Knockdown miR-223-3p reversed the inhibitory effect of circ_LRIG3 downregulation on progression of HCC cells}

To explore whether the functions of circ_LRIG3 was mediated by miR-223-3p, Hep3B and Huh7 cells were transfected with si-NC, si-circ_LRIG3, si-circ_LRIG3 + anti-miR-NC, or si-circ_LRIG3 + anti-miR-223-3p. As shown in Figure 4A-4D, the effects of si-circ_LRIG3 on promotion of G0/G1 phase cells and reduction of $S$ and $\mathrm{G} 2 / \mathrm{M}$ phases cells as well as cell proliferation were abolished by downregulating miR-223-3p. Moreover, the promotive effect of circ_LRIG3 knockdown on apoptosis was abated by downregulation of miR-223-3p (Figure 4E). Transwell assay indicated that interference of miR-223-3p reversed the inhibitory effects of circ_LRIG3 silence on migration and invasion (Figure 4F and 4G). Likewise, downregulating miR-223-3p also could abrogate the effects of si-circ_LRIG3 on decrease of snail expression and increase of E-cadherin expression in Hep3B and Huh7 cells (Figure 4H and 4I). Taken together, these findings disclosed that circ_LRIG3 knockdown inhibited the progression of HCC cell by upregulating miR-223-3p.

\section{MAP2K6 was a target gene of miR-223-3p in HCC cells}

To further elucidate the mechanism of miR-223-3p in HCC cells, target prediction was performed by TargetScan and MAP2K6 was identify as a candidate target for miR-223-3p (Figure 5A). To further whether MAP2K6 was a direct target of miR-223-3p, dual-luciferase reporter assay was carried out. We observed that the luciferase activity of MAP2K6-wt was markedly suppressed in cells transfected with miR-223-3p, but luciferase activity of MAP2K6-mut was not changed (Figure 5B and 5C). Transfection efficiency of miR-223-3p and anti-miR-223-3p was measured by qRT-PCR. Results showed that miR-223$3 p$ expression was increased in cells transfected with miR-223-3p while its expression was decreased in cells transfected with anti-miR-223-3p (Figure 5D), implying that miR-223-3p and anti-miR-223-3p were successfully transfected in Hep3B and Huh7 cells. Subsequently, the effect of miR-223-3p on expression of MAP2K6 was explored. The results from qRT-PCR and western blot analysis showed that overexpression of miR-223-3p reduced the MAP2K6 mRNA and protein expression while knockdown of miR-223-3p presented an opposite effect (Figure 5E and 5F). Next, the MAP2K6 mRNA and protein expression were examined by qRT-PCR and western blot assays in HCC cells and tissues. The results indicated that the mRNA and protein levels of MAP2K were overexpressed in HCC cells and tissues compared with their matched controls (Figure 5G-5J). In addition, we found that MAP2K6 mRNA expression was negatively correlated with miR-223-3p abundance (Figure 5K) ( $r=-0.5090, P=0.0003)$. 
Furthermore, we investigated whether circ_LRIG3 functioned as a molecular sponge of miR-223-3p to regulate the expression of MAP2K6. We observed that circ_LRIG3 deficiency decreased the mRNA and protein expression of MAP2K6 while interference of miR-223-3p reversed this effect (Figure $5 \mathrm{~L}$ and $5 \mathrm{M}$ ). Collectively, these data elaborated that circ_LRIG3 regulated MAP2K6 expression by sponging miR-223-3p in HCC cells.

\section{Overexpression of MAP2K6 abated the suppressive effect of si-circ_LRIG3 on progression of HCC cells}

To investigate whether MAP2K6 was involved in si-circ_LRIG3-mediated functions in HCC cells, Hep3B and Huh7 cells were transfected with si-NC, si-circ_LRIG3, si-circ_LRIG3 + pcDNA, or si-circ_LRIG3 + MAP2K6. As presented in Figure 6A and 6B, mRNA and protein expression of MAP2K6 were reduced in cells transfected with si-circ_LRIG3 compared to si-NC group, which was abated by addition of MAP2K6. Flow cytometry and MTT analysis showed that upregulation of MAP2K6 reversed the effects of sicirc_LRIG3 on promotion of G0/G1 phase cells and reduction of $S$ and G2/M phases cells as well as cell proliferation (Figure 6C-6F). Additionally, overexpression of MAP2K6 abolished the pro-apoptosis, antimigration and anti-invasion effects caused by silencing circ_LRIG3 (Figure 6G-6I). Western blot assay proved that co-transfection of MAP2K6 attenuated the suppression of snail expression and promotion of E-cadherin expression in Hep3B and Huh7 cells transfected with si-circ_LRIG3 (Figure 6J and 6K). Therefore, we concluded that circ_LRIG3 knockdown suppressed the progression of HCC cell by downregulating MAP2K6.

\section{Silencing circ_LRIG3 inhibited the activation of MAPK/ERK pathway through upregulating miR-223-3p and downregulating MAP2K6}

MAPK/ERK signaling pathway is known to be activated in many cancers [17]. MAPK/ERK-related proteins were analyzed by western blot assay. Results demonstrated that knockdown of circ_LRIG3 reduced the protein levels of $p$-MAPK and $p$-ERKs, which was reversed by interference of miR-223-3p or overexpression of MAP2K6, but we observed no change of total MAPK and ERKs protein in Hep3B and Huh7 cells (Figure 7A and 7B). These findings indicated that circ_LRIG3 modulated the MAPK/ERK pathway by affecting miR-223-3p and MAP2K6 expression.

\section{Knockdown of circ_LRIG3 limited tumor growth by regulating miR-223-3p and MAP2K6 expression}

Sh-NC or sh-circ_LRIG3-transfected Huh7 cells were introduced into nude mice to assess the role of circ_LRIG3 in vivo. As displayed in Figure 8A and 8B, interference of circ_LRIG3 reduced tumor volume and weight in xenograft model. We then detected the expression of circ_LRIG3, miR-223-3p and MAP2K6 in tumor tissues. As shown in Figure 8C-8E, silencing circ_LRIG3 decreased the expression of circ_LRIG3 and MAP2K6 while elevated the abundance miR-223-3p in excised tumor masses. Western blot assay also proved that circ_LRIG3 interference led to decrease of MAP2K6 protein expression in tumor tissues (Figure 8F). To sum up, these results disclosed that circ_LRIG3 deficiency inhibited tumor growth via upregulating miR-223-3p and downregulating MAP2K6 in vivo. 


\section{Discussion}

HCC is one of the most common deadly cancers in the world. Growing evidence showed that the abnormal expression of circRNAs was tightly related to tumorigenesis and development of tumors, including HCC [18]. Hence, more efforts should be made to deeply explain the functional roles and underlying mechanisms of circ_LRIG3 in HCC. Here, we found that circ_LRIG3 knockdown inhibited the progression of HCC by regulating miR-223-3p/MAP2K6 axis and inactivating MAPK/ERK signaling pathway.

Accumulating evidence has shown that circRNAs are abundant in eukaryotes and aberrantly expressed in human cancers [19]. In addition, because of their covalently closed-structure, circRNAs are more stable and more suitable as efficacious biomarkers than linear-RNAs, such as IncRNAs and miRNAs [20]. For instance, circ_UVRAG [21], circ_BACH2 [22] and circ_ANKS1B [23] have been identified to be diagnostic or prognostic biomarker for gastric cancer, papillary thyroid carcinoma and breast cancer, respectively. Previous report has been demonstrated that hsa_circ_0027345 (a circRNA derived from linear LRIG3) was overexpressed in HCC tissues [11]. However, there is no report on the functions and underlying mechanism of circ_LRIG3 in HCC. Consistent with previous report, we also uncovered that circ_LRIG3 level was enhanced in HCC tissues and cell lines. Additionally, we observed that knockdown of circ_LRIG3 inhibited the progression of $\mathrm{HCC}$ cells via reducing cell proliferation, metastasis and promoting apoptosis. These findings suggested that circ_LRIG3 might act as a tumor promoter in HCC.

Emerging evidence showed that some circRNAs participated in tumorigenesis through functioning as sponges for miRNAs [16, 24]. Then, circinteractome was utilized to predict the potential targets of circ_LRIG3, the data showed that circ_LRIG3 might interact with miR-223-3p, which was validated using the dual-luciferase reporter assay in HCC cells. MiR-223 a well-studied miRNA, presented different properties in different cancers, acting as an oncogene in colorectal cancer [25], gastric cancer [26] and prostate cancer [27], or as an anti-oncogene in esophageal carcinoma [28], breast cancer [29] and osteosarcoma [30]. Previous studies have suggested that miR-223 was lowly expressed HCC [31, 32]. Moreover, miR-223 has been suggested to repress HCC cell growth and accelerate apoptosis through the Rab1-mediated mTOR activation [33]. In agreement with these findings, we proved that miR-223-3p abundance was reduced in HCC tissues and cells, and its interference abated the repressive impact of circ_LRIG3 downregulation on progression of HCC cells. These data suggested that circ_LRIG3 exerted its functions by sponging miR-223-3p in HCC cells.

It is well known that miRNAs mediate various cellular activities through regulating their molecular targets [34]. Thus, the possible downstream targets of miR-223-3p were searched through the TargetScan software. Our results revealed that MAP2K6 was a direct target of miR-223-3p. MAP2K6 (important components of MAPK signal pathway) is involved in a variety of physiological and pathological processes as well as drug resistance in human cancer cells, and it has been recognized as an oncogene in many cancers, such as esophageal adenocarcinoma [35], prostate cancer [36] and colon cancers [15]. However, the expression and effect of MAP2K6 in HCC cells have not been clarified. Here, it was found 
that the MAP2K6 was overexpressed in HCC tissues and cells, and was positively regulated by circ_LRIG3 and inversely modulated by miR-223-3p. Functional experiments displayed that upregulation of MAP2K6 abolished the suppressive effect of circ_LRIG3 interference on progression of HCC cells. Moreover, in vivo experiments presented that circ_LRIG3 silence inhibited tumor growth through upregulating miR-223-3p and downregulating MAP2K6 expression. Collectively, our results disclosed that circ_LRIG3 knockdown repressed HCC progression by regulating miR-223-3p/MAP2K6 axis.

Previous studies show that HCC is associated with elevated expression and activity of MAPK signaling intermediates (ie, MEK, ERK) [37]. Moreover, activation of MAPK/ERK signaling pathway predicted poor prognosis in HCC, and many anticancer agents exerted their effects by blocking MAPK/ERK pathway [38, 39]. These findings suggested that MAPK/ERK signaling pathway played key roles in HCC progression. In our research, results proved that circ_LRIG3 downregulation repressed the activation of MAPK/ERK signaling pathway through upregulating miR-223-3p and downregulating MAP2K6.

\section{Conclusions}

In summary, we demonstrated that circ_LRIG3 and MAP2K6 were overexpressed and miR-223-3p abundance was reduced in HCC tissues and cells. Circ_LRIG3 interference limited cell growth and metastasis but facilitated apoptosis in HCC cells through regulating miR-223-3p/MAP2K6 axis and inactivating MAPK/ERK signaling pathway. These findings might offer novel targets for treatment and prediction of HCC.

\section{Declarations}

\section{Ethics approval and consent to participate}

The study was approved by the Ethics Committee of Laiyang Central Hospital Of Yantai City and written informed consents were collected from all patients and hospitals.

The animal experiment was permitted by the Animal Research Committee of Laiyang Central Hospital Of Yantai

City and performed in accordance with the guidelines of the National Animal Care and Ethics Institution.

\section{Consent for publication}

Not applicable

\section{Availability of data and materials}

The data sets used and/or analyzed during the current study are available from the corresponding author on reasonable request.

\section{Competing interests}


The authors declare that they have no conflicts of interest.

\section{Funding}

None

\section{Authors' contributions}

HS and JZ performed experiments, analyzed data, and wrote the manuscript. LZ designed research, performed experiments, and analyzed data. YC conceived and designed research.

\section{Acknowledgment}

Thanks for all participants involved in this study.

\section{References}

1. Torre LA, Bray F, Siegel RL, Ferlay J, Lortet-Tieulent J, Jemal A. Global cancer statistics, 2012. CA Cancer J Clin. 2015;65(2):87-108.

2. Jinjuvadia R, Salami A, Lenhart A, Jinjuvadia K, Liangpunsakul S, Salgia R. Hepatocellular carcinoma: a decade of hospitalizations and financial burden in the United States. Am J Med Sci. 2017;354(4):362-9.

3. Altekruse SF, McGlynn KA, Reichman ME. Hepatocellular carcinoma incidence, mortality, and survival trends in the United States from 1975 to 2005. J Clin Oncol. 2009;27(9):1485.

4. Li X, Yang L, Chen L-L. The biogenesis, functions, and challenges of circular RNAs. Mol Cell. 2018;71(3):428-42.

5. Memczak S, Jens M, Elefsinioti A, Torti F, Krueger J, Rybak A, et al. Circular RNAs are a large class of animal RNAs with regulatory potency. Nature. 2013;495(7441):333.

6. Chen L-L, Yang L. Regulation of circRNA biogenesis. RNA Biol. 2015;12(4):381-8.

7. Qin M, Liu G, Huo X, Tao X, Sun X, Ge Z, et al. Hsa_circ_0001649: a circular RNA and potential novel biomarker for hepatocellular carcinoma. Cancer Biomark. 2016;16(1):161-9.

8. Meng S, Zhou H, Feng Z, Xu Z, Tang Y, Li P, et al. CircRNA: functions and properties of a novel potential biomarker for cancer. Mol Cancer. 2017;16(1):94.

9. Yu L, Gong X, Sun L, Zhou Q, Lu B, Zhu L. The circular RNA Cdr1 as act as an oncogene in hepatocellular carcinoma through targeting miR-7 expression. PLoS One. 2016;11(7).

10. Yu J, Xu QG, Wang ZG, Yang Y, Zhang L, Ma JZ, et al. Circular RNA cSMARCA5 inhibits growth and metastasis in hepatocellular carcinoma. J Hepatol. 2018;68(6):1214-27.

11. Sun S, Wang W, Luo X, Li Y, Liu B, Li X, et al. Circular RNA circ-ADD3 inhibits hepatocellular carcinoma metastasis through facilitating EZH2 degradation via CDK1-mediated ubiquitination. Am J Cancer Res. 2019;9(8):1695. 
12. Hansen TB, Jensen TI, Clausen BH, Bramsen JB, Finsen B, Damgaard CK, et al. Natural RNA circles function as efficient microRNA sponges. Nature. 2013;495(7441):384-8.

13. Ardekani AM, Naeini MM. The role of microRNAs in human diseases. Avicenna J Med Biotechnol. 2010;2(4):161.

14. Zhang C, Zhang J. Decreased expression of microRNA-223 promotes cell proliferation in hepatocellular carcinoma cells via the insulin-like growth factor-1 signaling pathway. Exp Ther Med. 2018;15(5):4325-31.

15. Parray AA, Baba RA, Bhat HF, Wani L, Mokhdomi TA, Mushtaq U, et al. MKK6 is upregulated in human esophageal, stomach, and colon cancers. Cancer Invest. 2014;32(8):416-22.

16. Kulcheski FR, Christoff AP, Margis R. Circular RNAs are miRNA sponges and can be used as a new class of biomarker. J Biotechnol. 2016;238:42-51.

17. Roy SK, Srivastava RK, Shankar S. Inhibition of PI3K/AKT and MAPK/ERK pathways causes activation of FOXO transcription factor, leading to cell cycle arrest and apoptosis in pancreatic cancer. J Mol Signal. 2010;5(1):10.

18. Bhan A, Soleimani M, Mandal SS. Long noncoding RNA and cancer: a new paradigm. Cancer Res. 2017;77(15):3965-81.

19. Shang Q, Yang Z, Jia R, Ge S. The novel roles of circRNAs in human cancer. Mol Cancer. 2019;18(1):1-10.

20. Wang Y, Mo Y, Gong Z, Yang X, Yang M, Zhang S, et al. Circular RNAs in human cancer. Mol Cancer. 2017;16(1):25.

21. Yang $C$, Wu S, Wu X, Zhou X, Jin S, Jiang H. Silencing circular RNA UVRAG inhibits bladder cancer growth and metastasis by targeting the microRNA-223/fibroblast growth factor receptor 2 axis. Cancer Sci. 2019;110(1):99-106.

22. Cai X, Zhao Z, Dong J, Lv Q, Yun B, Liu J, et al. Circular RNA circBACH2 plays a role in papillary thyroid carcinoma by sponging miR-139-5p and regulating LMO4 expression. Cell Death Dis. 2019;10(3):1-12.

23. Zeng K, He B, Yang BB, Xu T, Chen X, Xu M, et al. The pro-metastasis effect of circANKS1B in breast cancer. Mol Cancer. 2018;17(1):1-19.

24. Subramanian S. Competing endogenous RNAs (ceRNAs): new entrants to the intricacies of gene regulation. Front Genet. 2014;5:8.

25. Li ZW, Yang YM, Du LT, Dong Z, Wang LL, Zhang X, et al. Overexpression of miR-223 correlates with tumor metastasis and poor prognosis in patients with colorectal cancer. Med Oncol. 2014;31(11):256.

26. Li J, Guo Y, Liang X, Sun M, Wang G, De W, et al. MicroRNA-223 functions as an oncogene in human gastric cancer by targeting FBXW7/hCdc4. J Cancer Res Clin Oncol. 2012;138(5):763-74.

27. Wei Y, Yang J, Yi L, Wang Y, Dong Z, Liu Z, et al. MiR-223-3p targeting SEPT6 promotes the biological behavior of prostate cancer. Sci Rep. 2014;4:7546. 
28. Li S, Li Z, Guo F, Qin X, Liu B, Lei Z, et al. miR-223 regulates migration and invasion by targeting Artemin in human esophageal carcinoma. J Biomed Sci. 2011;18(1):24.

29. Yang Y, Jiang Z, Ma N, Wang B, Liu J, Zhang L, et al. MicroRNA-223 targeting STIM1 inhibits the biological behavior of breast cancer. Cell Physiol Biochem. 2018;45(2):856-66.

30. Ji Q, Xu X, Song Q, Xu Y, Tai Y, Goodman SB, et al. miR-223-3p inhibits human osteosarcoma metastasis and progression by directly targeting CDH6. Mol Ther. 2018;26(5):1299-312.

31. Karakatsanis A, Papaconstantinou I, Gazouli M, Lyberopoulou A, Polymeneas G, Voros D. Expression of microRNAs, miR-21, miR-31, miR-122, miR-145, miR-146a, miR-200c, miR-221, miR-222, and miR223 in patients with hepatocellular carcinoma or intrahepatic cholangiocarcinoma and its prognostic significance. Mol Carcinog. 2013;52(4):297-303.

32. Bhattacharya S, Steele R, Shrivastava S, Chakraborty S, Di Bisceglie AM, Ray RB. Serum miR-30e and miR-223 as novel noninvasive biomarkers for hepatocellular carcinoma. Am J Pathol. 2016;186(2):242-7.

33. Dong Z, Qi R, Guo X, Zhao X, Li Y, Zeng Z, et al. MiR-223 modulates hepatocellular carcinoma cell proliferation through promoting apoptosis via the Rab1-mediated mTOR activation. Biochem Biophys Res Commun. 2017;483(1):630-7.

34. Felekkis K, Touvana E, Stefanou C, Deltas C. microRNAs: a newly described class of encoded molecules that play a role in health and disease. Hippokratia. 2010;14(4):236.

35. Lin S, Liu K, Zhang Y, Jiang M, Lu R, Folts CJ, et al. Pharmacological targeting of p38 MAP-Kinase 6 (MAP2K6) inhibits the growth of esophageal adenocarcinoma. Cell Signal. 2018;51:222-32.

36. Lotan T, Lyon M, Huo D, Taxy J, Brendler C, Foster B, et al. Up-regulation of MKK4, MKK6 and MKK7 during prostate cancer progression: an important role for SAPK signalling in prostatic neoplasia. $\mathrm{J}$ Pathol. 2007;212(4):386-94.

37. Wiesenauer CA, Yip-Schneider MT, Wang Y, Schmidt CM. Multiple anticancer effects of blocking MEKERK signaling in hepatocellular carcinoma. J Am Coll Surg. 2004;198(3):410-21.

38. Schmitz KJ, Wohlschlaeger J, Lang H, Sotiropoulos GC, Malago M, Steveling K, et al. Activation of the ERK and AKT signalling pathway predicts poor prognosis in hepatocellular carcinoma and ERK activation in cancer tissue is associated with hepatitis C virus infection. J Hepatol. 2008;48(1):83-90.

39. Liang R-R, Zhang S, Qi J-A, Wang Z-D, Li J, Liu P-J, et al. Preferential inhibition of hepatocellular carcinoma by the flavonoid Baicalein through blocking MEK-ERK signaling. Int $\mathrm{J}$ Oncol. 2012;41(3):969-78.

\section{Figures}



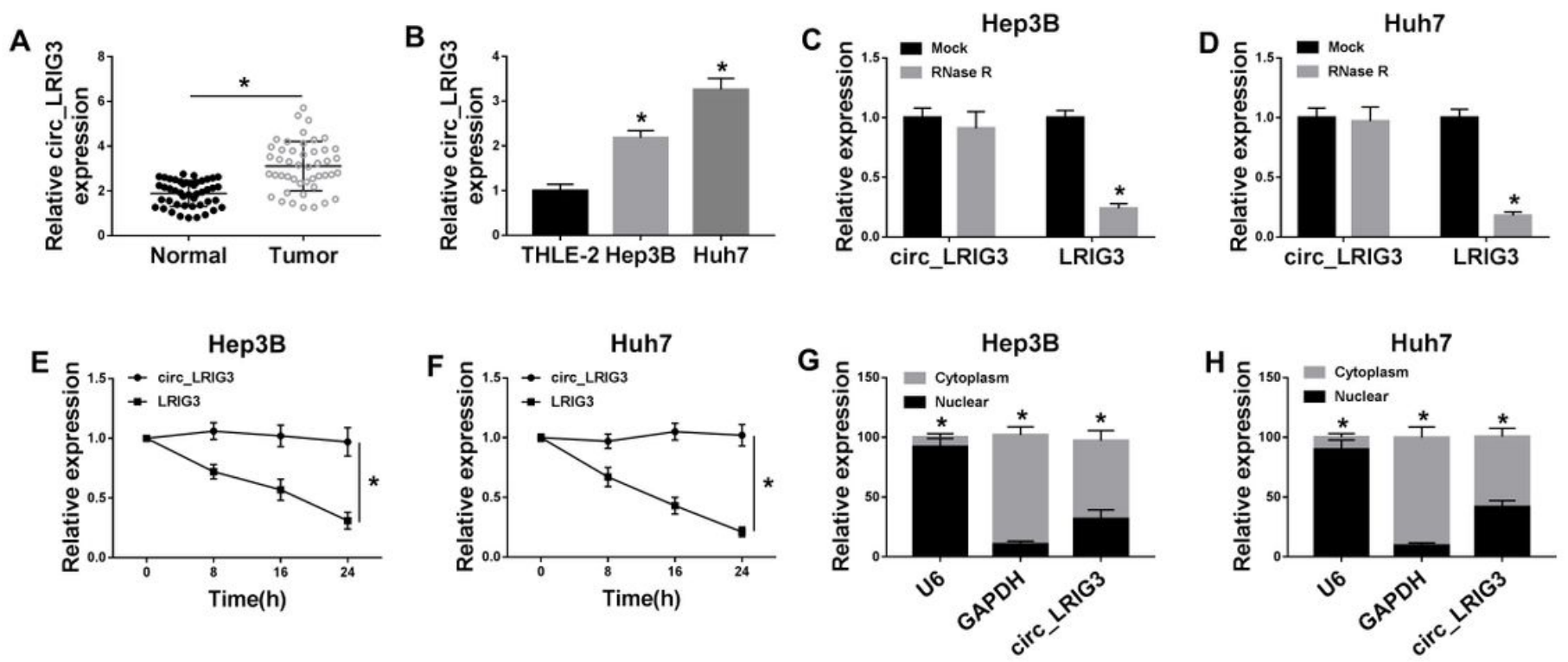

\section{Figure 1}

The expression of circ_LRIG3 was enhanced in HCC tissues and cells. (A) The expression of circ_LRIG3 was determined in 46 pairs of HCC tissues and normal tissues using qRT-PCR analysis. (B) The level of circ_LRIG3 was measured by qRT-PCR in HCC cells (Hep3B and Huh7) and human normal liver cells (THLE-2). (C-F) The relative levels of circ_LRIG3 and LRIG3 were determined after treatment with RNase R or actinomycin D by qRT-PCR in Hep3B and Huh7 cells. ( $G$ and $H$ ) The qRT-PCR assay determined the subcellular location of circ_LRIG3 in Hep3B and Huh7 cells. ${ }^{*} P<0.05$. 

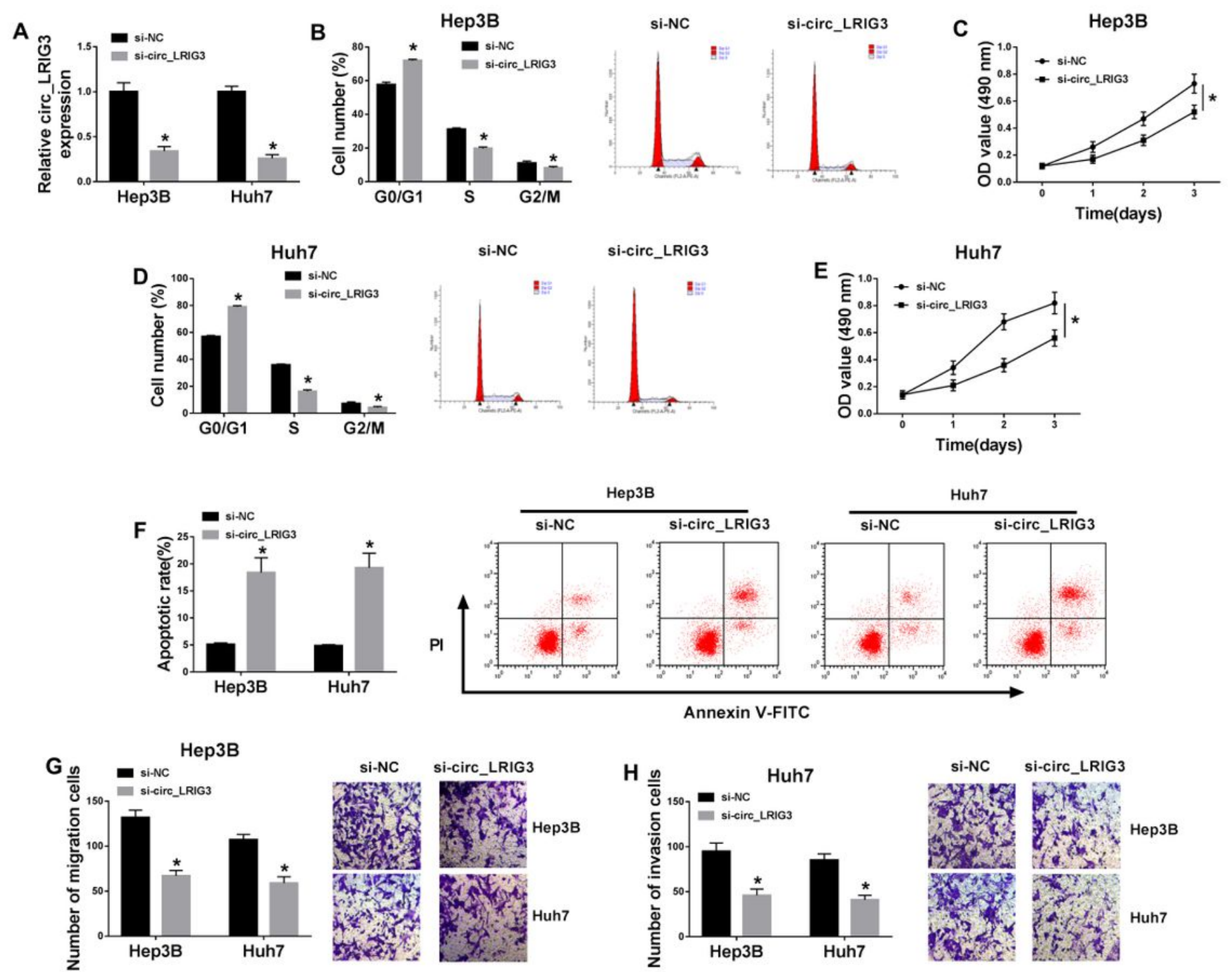

Hep3B
si-circ_LRIG3
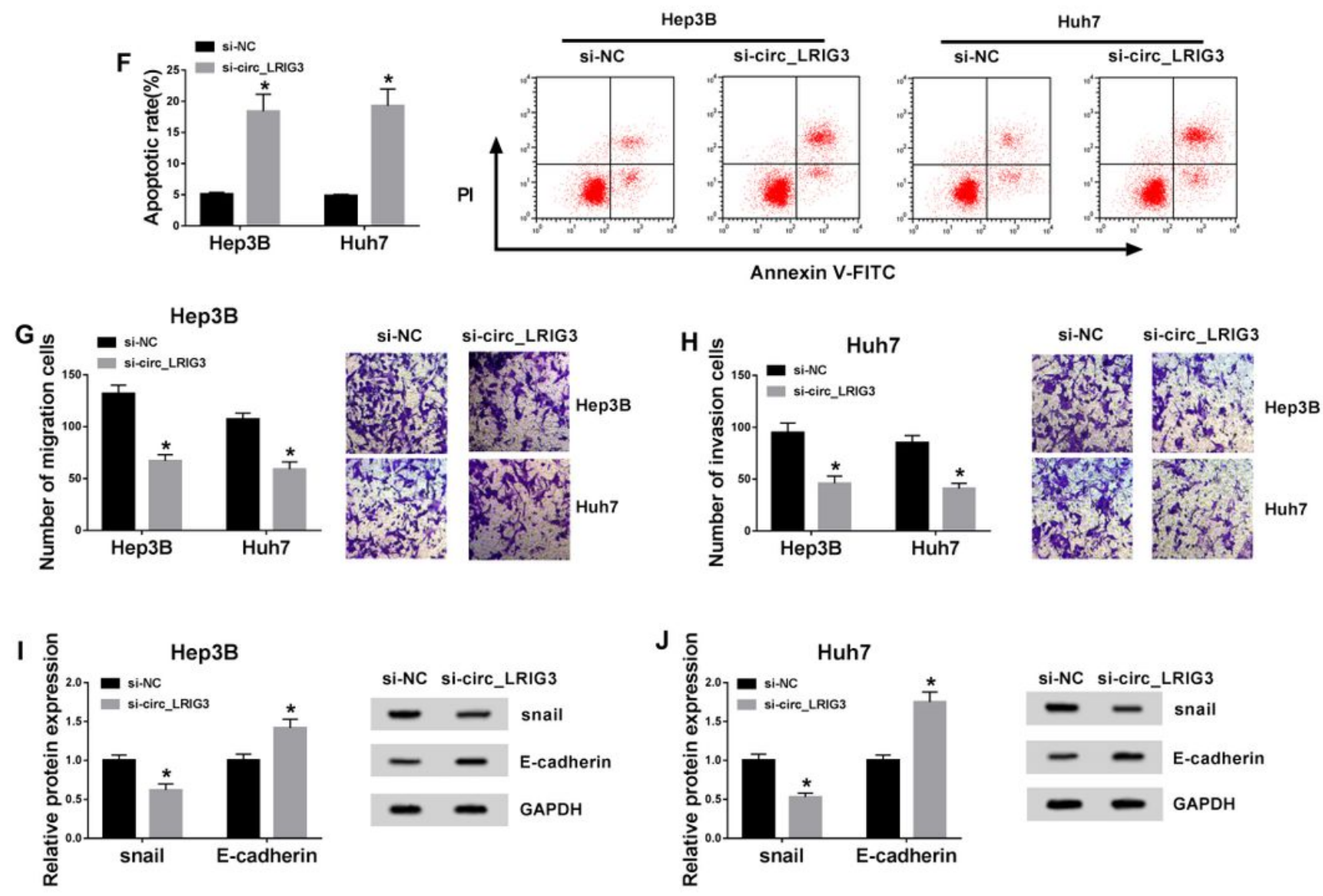

Figure 2

Knockdown of circ_LRIG3 inhibited the progression of HCC cells through inhibiting cell proliferation and metastasis and promoting apoptosis. Hep3B and Huh7 cells were transfected with si-NC or si-circ_LRIG3. (A) The expression of circ_LRIG3 was analyzed by qRT-PCR. (B and D) Cell cycle distribution was analyzed using the flow cytometry. (C and E) MTT assay was utilized to assess cell proliferation. ( $F$ ) Cell apoptosis was examined using flow cytometry analysis. ( $\mathrm{G}$ and $\mathrm{H}$ ) Transwell assay was used to 
determine cell migration and invasion abilities. ( $\mathrm{I}$ and $\mathrm{J})$ The protein levels of snail and E-cadherin were evaluated by western blot assay. ${ }^{*}<0.05$.
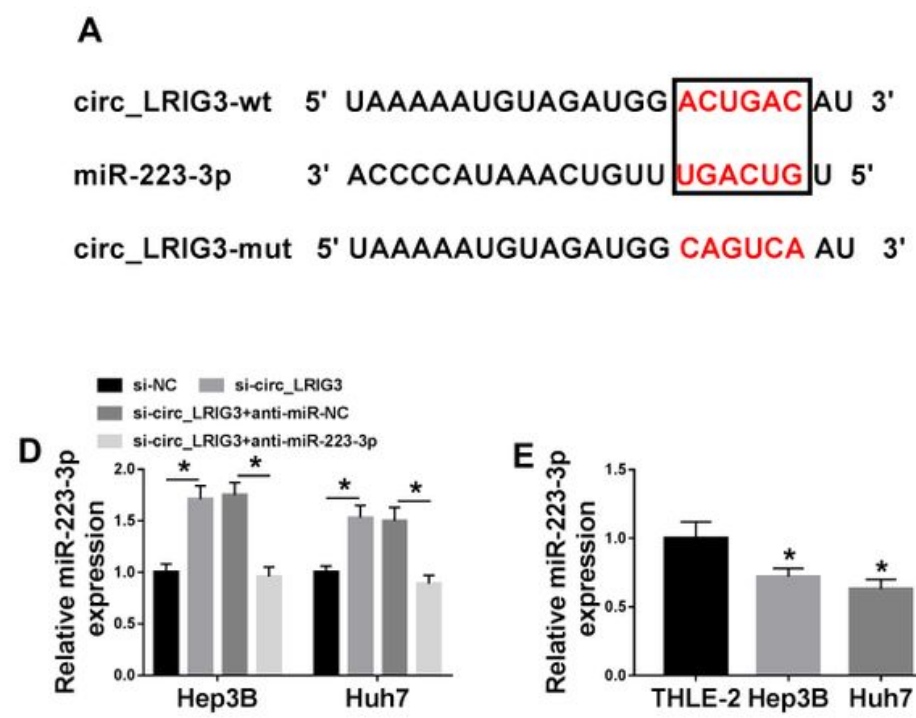
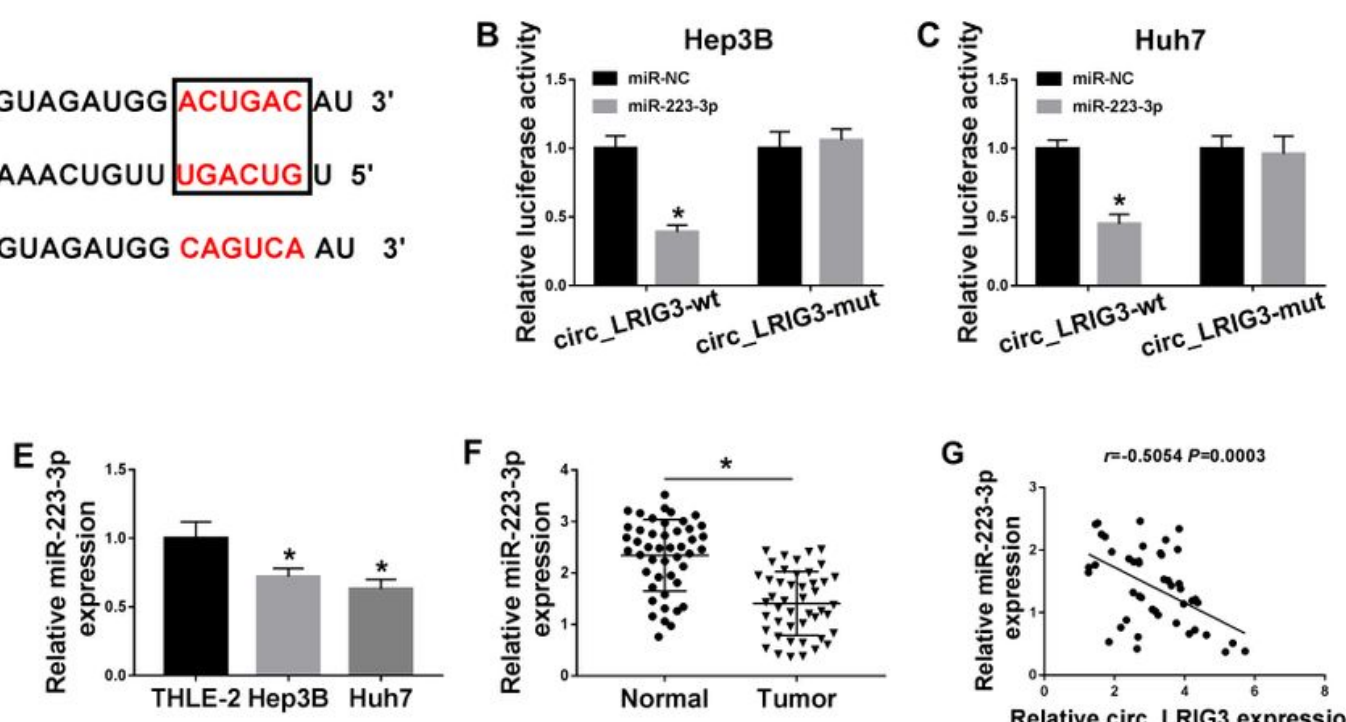
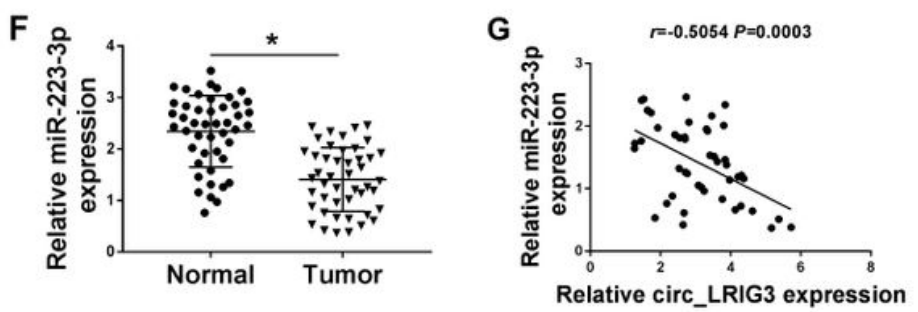

\section{Figure 3}

Circ_LRIG3 could interact with miR-223-3p in HCC cells. (A) The putative binding sites between circ_LRIG3 and miR-223-3p were predicted by circinteractome tool. (B and C) Dual-luciferase luciferase reporter assay was utilized to detect the luciferase activity in Hep3B and Huh7 cells co-transfected with circ_LRIG3-wt or circ_LRIG3-mut and miR-NC or miR-223-3p mimic. (D) The expression of miR-223-3p was measured by qRT-PCR in Hep3B and Huh7 cells transfected with si-NC, si-circ_LRIG3, si-circ_LRIG3 + antimiR-NC, or si-circ_LRIG3 + anti-miR-223-3p. (E and F) The abundance of miR-223-3p was analyzed by qRT-PCR in HCC cells (Hep3B and Huh7), HCC tissues and their matched controls. (G) The correlation between miR-223-3p abundance and circ_LRIG3 level was analyzed in HCC tissues. ${ }^{*} P<0.05$. 
Нер3в

- si-NC

si-circ_LRIG3+anti-miR-NC

A si-circ_LRIG3+anti-miR-223-3p

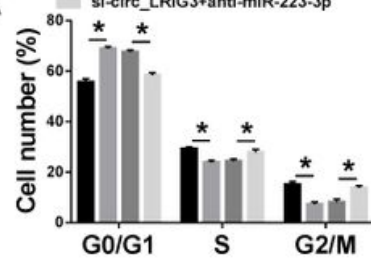

si-NC si-circ_LRIG3

E

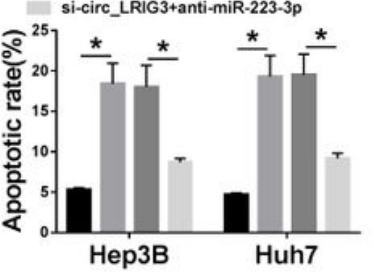

Hep3B

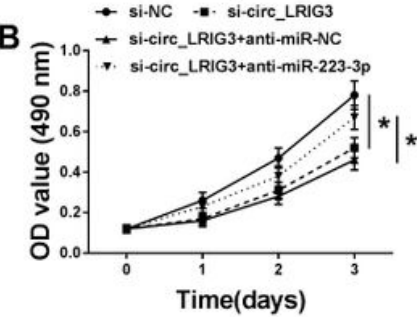

Huh7

C

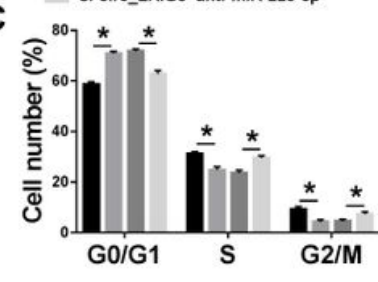

Huh7

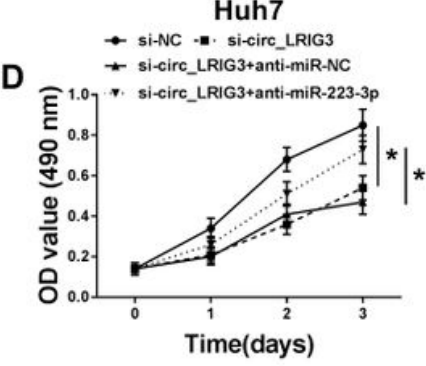

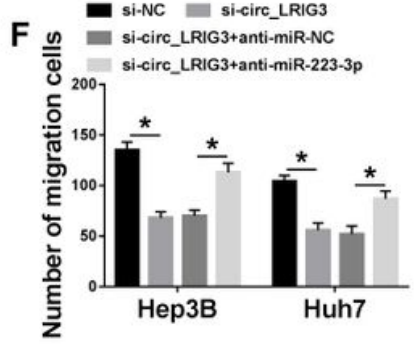

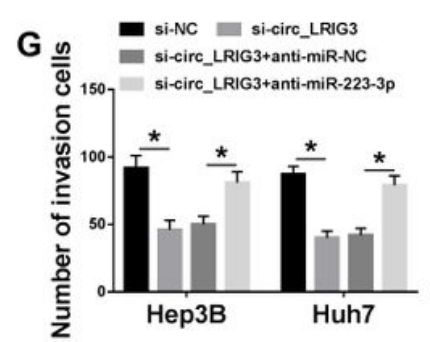

Huh7
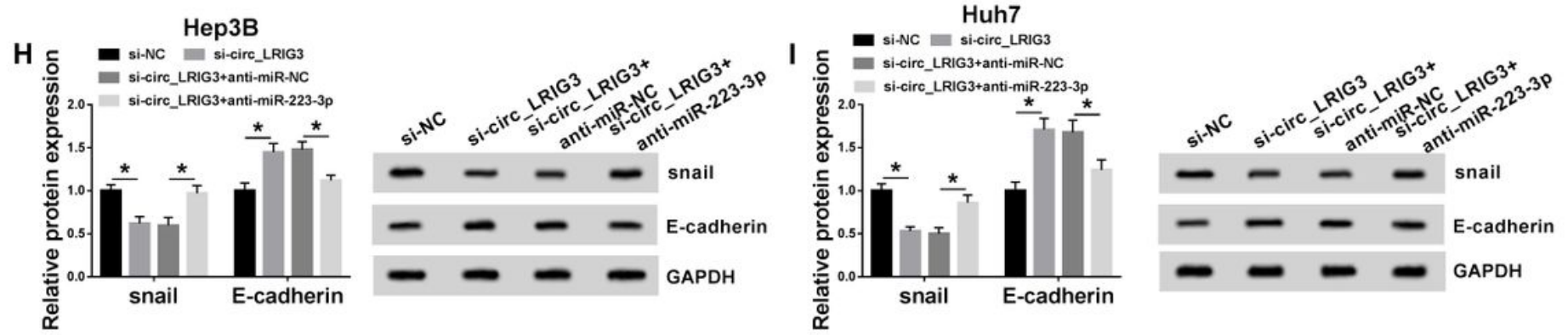

Figure 4

. Inhibition of miR-223-3p reversed the regulatory effect of circ_LRIG3 interference on progression of HCC cells. Hep3B and Huh7 cells were transfected with si-NC, si-circ_LRIG3, si-circ_LRIG3 + anti-miR-NC, or sicirc_LRIG3 + anti-miR-223-3p. (A and C) Cell cycle distribution was determined by flow cytometry. (B and D) MTT assay was conducted to evaluate cell proliferation. (E) Cell apoptosis was measured by flow cytometry analysis. ( $F$ and $\mathrm{G}$ ) Transwell assay was employed to detect the number of migrated and invaded cells. ( $\mathrm{H}$ and I) Western blot analysis was applied to determine the protein levels of snail and Ecadherin. ${ }^{*} \mathrm{P}<0.05$. 
A

MAP2K6 3' UTR

MAP2K6-wT 5' UUGUGUCAAUCUACA AACUGAC 3' miR-223-3p 3' AccCCAUAAACUgU UUgacug U $5^{\prime}$

MAP2K6-mut 5' UUGUGUCAAUCUACA CCAGUCA U 3'
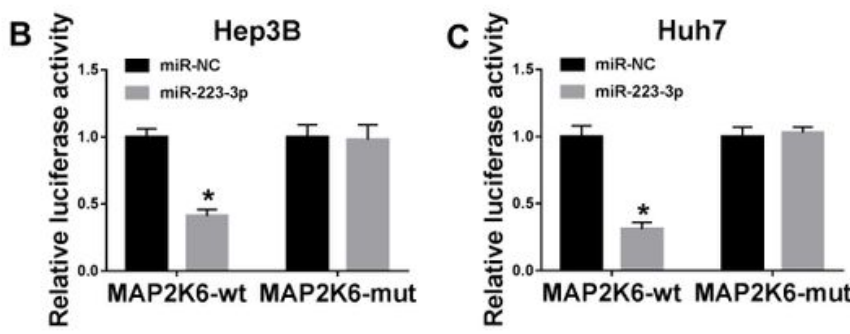
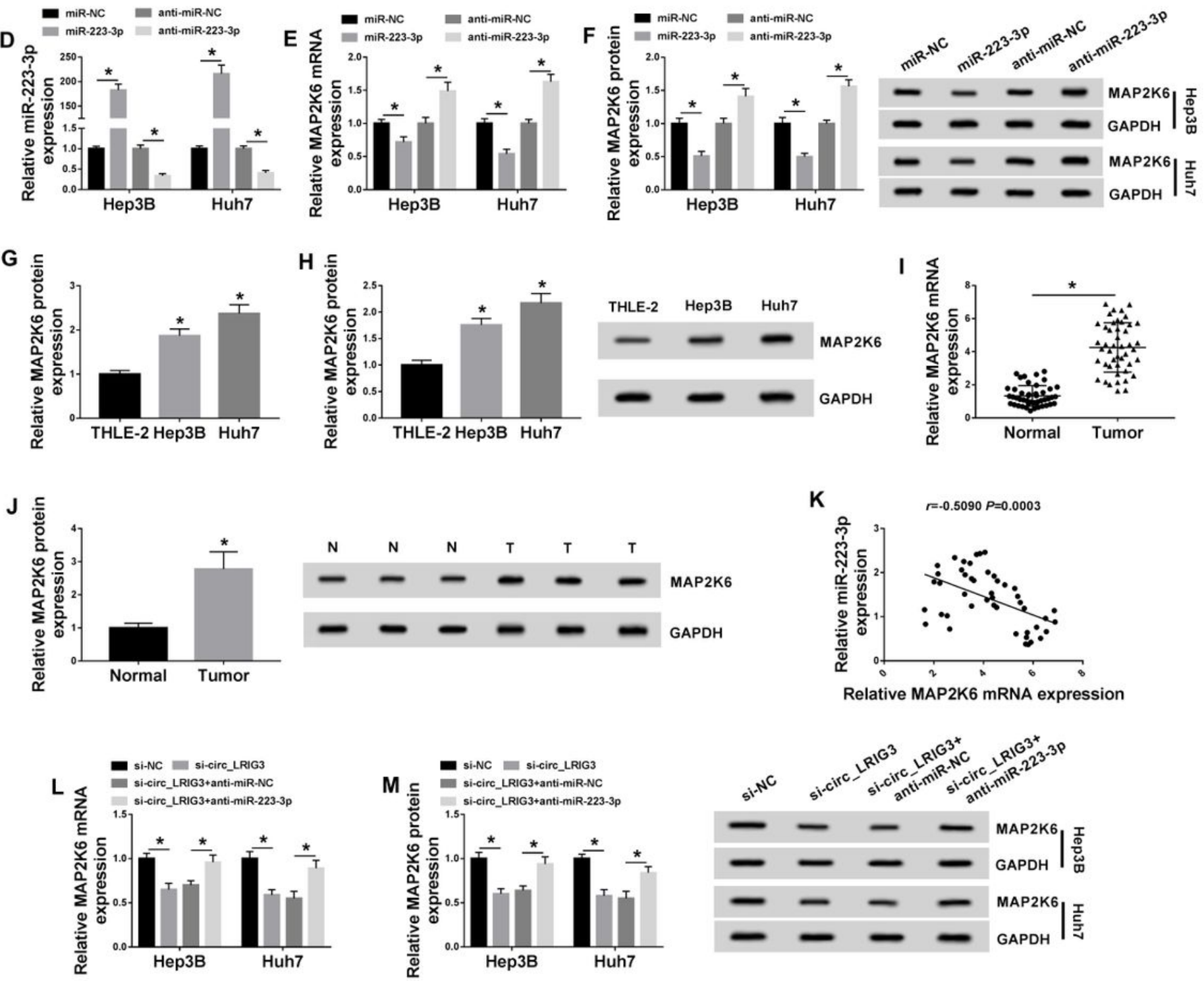

\section{Figure 5}

MAP2K6 was targeted by miR-223-3p in HCC cells. (A) The putative binding sequence of miR-223-3p in the 3'UTR of MAP2K6 was predicted by TargetScan. (B and C) Relative luciferase activity was determined in Hep3B and Huh7 cells co-transfected with MAP2K6-wt or MAP2K6-mut and miR-NC or or miR-223-3p mimic. (D) Relative miR-223-3p expression was measured by qRT-PCR in Hep3B and Huh7 cells transfected with miR-NC, miR-223-3p, anti-miR-NC, or anti-miR-223-3p. (E and F) MAP2K6 mRNA or protein expression was analyzed by qRT-PCR or western blot assays in Hep3B and Huh7 cells transfected 
with miR-NC, miR-223-3p, anti-miR-NC, or anti-miR-223-3p. ( $G$ and H) The mRNA and protein levels of MAP2K6 were examined in HCC cells (Hep3B and Huh7) and THLE-2 cells by qRT-PCR and western blot analyses, respectively. (I and J) QRT-PCR and western blot assays were conducted to measure the mRNA and protein levels of MAP2K6 in HCC tissues and normal tissues, respectively. (K) The association between miR-223-3p abundance and MAP2K6 mRNA level was analyzed in HCC tissues. ( $L$ and M) The mRNA and protein levels of MAP2K6 were detected in Hep3B and Huh7 cells transfected with si-NC, sicirc_LRIG3, si-circ_LRIG3 + anti-miR-NC, or si-circ_LRIG3 + anti-miR-223-3p by qRT-PCR and western blot analyses, respectively. ${ }^{*} \mathrm{P}<0.05$.
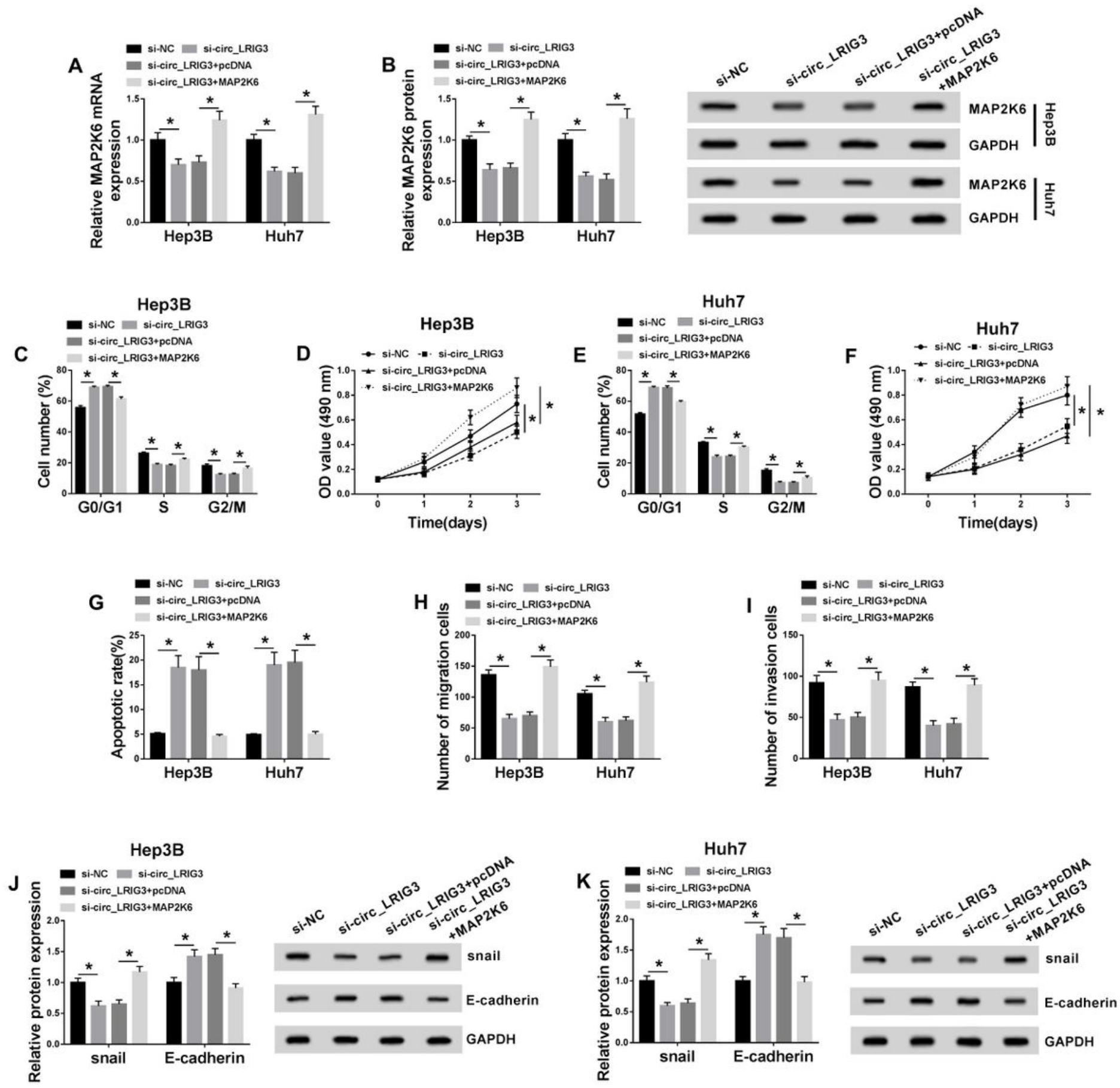

Figure 6 
Interference of circ_LRIG3 suppressed the progression of HCC cells by downregulating MAP2K6. Hep3B and Huh7 cells were transfected with si-NC, si-circ_LRIG3, si-circ_LRIG3 + pcDNA, or si-circ_LRIG3 + MAP2K6. (A and B) The mRNA and protein levels of MAP2K6 were measured by qRT-PCR and western blot analyses, respectively. ( $C$ and E) Flow cytometry was applied to determine the cell cycle distribution. (D and F) Cell proliferation was assessed by MTT analysis. (G) Cell apoptosis was determined using flow cytometry analysis. ( $\mathrm{H}$ and I) Transwell assay was employed to count the number of migrated or invaded cells. ( $\mathrm{J}$ and $\mathrm{K}$ ) The protein levels of snail and E-cadherin were tested by western blot analysis. ${ }^{*} \mathrm{P}<0.05$.
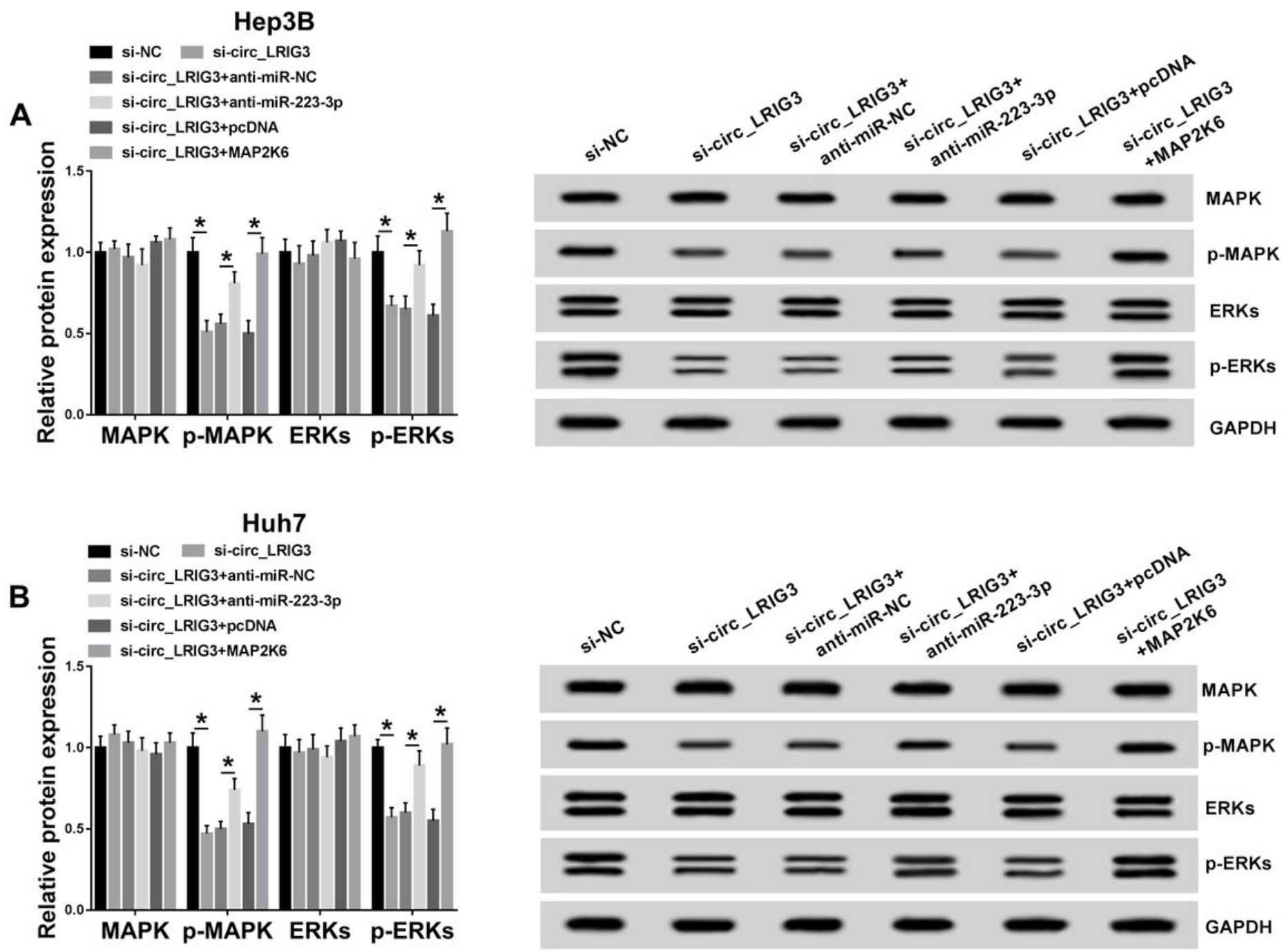

\section{Figure 7}

Downregulation of circ_LRIG3 suppressed the activation of MAPK/ERK pathway by regulating miR-223$3 p$ and MAP2K6 expression. ( $A$ and $B$ ) The protein levels of MAPK, p-MAPK, ERKs, and p-ERKs were examined by western blot analysis in Hep3B and Huh7 cells transfected with si-NC, si-circ_LRIG3, sicirc_LRIG3 + anti-miR-NC, si-circ_LRIG3 + anti-miR-223-3p, si-circ_LRIG3 + pcDNA, or si-circ_LRIG3 + MAP2K6. *P<0.05. 

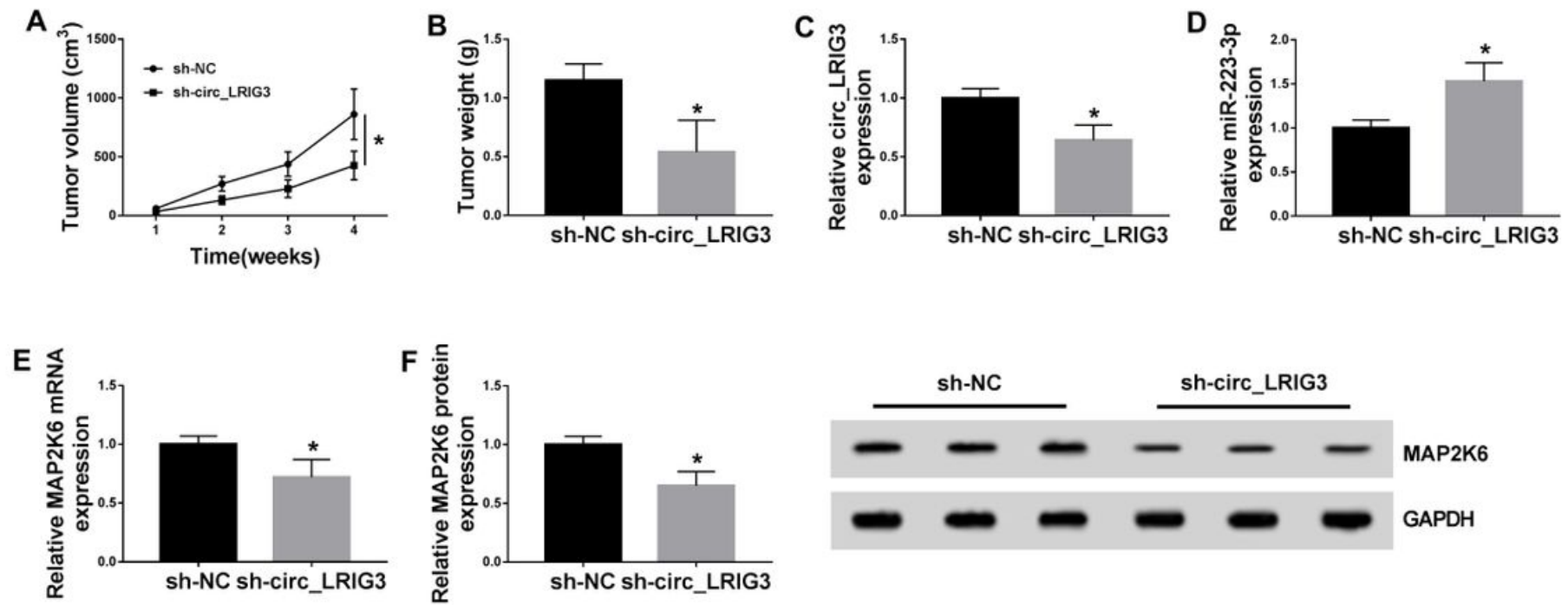

\section{Figure 8}

Silence of circ_LRIG3 repressed tumor growth by upregulating miR-223-3p and downregulating MAP2K6. Sh-NC or sh-circ_LRIG3-transfected Huh7 cells were introduced into nude mice to establish mice model. ( $A$ and B) Tumor volume and weight were examined. (C-E) The expression levels of circ_LRIG3, miR-223$3 p$ and MAP2K6 were determined by qRT-PCR in tumor tissues. $(F)$ Western blot assay was applied to analyze the protein expression of MAP2K6 in tumor tissues. ${ }^{*} \mathrm{P}<0.05$. 